\title{
CONOCIMIENTO DEL MERCADO DE VALORES EN LAS MEDIANAS Y GRANDES EMPRESAS DEL DEPARTAMENTO DE BOYACÁ: DIAGNÓSTICO Y PROPUESTA ESTRATÉGICA ${ }^{1}$
}

\author{
MARKET KNOWLEDGE IN MEDIUM AND LARGE COMPANIES IN BOYACA \\ DEPARTMENT: DIAGNOSIS AND STRATEGIC APPROACH
}

\author{
Gina Paola Fonseca Cifuentes** \\ Lady Johanna Castaño Buitrago***
}

Para citar este artículo: Fonseca, G. y Castaño, L. (2014). "Conocimiento del mercado de valores en las medianas y grandes empresas del departamento de Boyacá: diagnóstico y propuesta estratégica”. Inquietud Empresarial. Vol. XIV (2), p.p. 11-29

Fecha de recepción: 25 de septiembre de 2014 Fecha de Aceptación: 26 de noviembre de 2014

\footnotetext{
1 Este artículo es resultado del proyecto de investigación denominado "Estudio de Percepción del Mercado de Valores en las Medianas y Grandes Empresas del departamento de Boyacá", desarrollado por el Grupo de Investigación Gerencia del valor y finanzas, adscrito a la Escuela de Administración de Empresas de la UPTC Tunja.

${ }^{* *}$ Administradora de Empresas, Especialista en Finanzas de la UPTC y Magister en Administración Económica y Financiera de la Universidad Tecnológica de Pereira. Docente ocasional de la Escuela de Administración de Empresas de la UPTC. Correo electrónico:gfonseca123@gmail.com

${ }^{* * \star A d m i n i s t r a d o r a ~ d e ~ E m p r e s a s ~ d e ~ l a ~ U P T C . ~ C o r r e o ~ e l e c t r o ́ n i c o: ~ c a s l a d y 05 @ g m a i l . c o m ~}$
} 


\title{
RESUMEN
}

El presente estudio es abordado desde la perspectiva analítica y estratégica en el área de las finanzas empresariales, buscando determinar el nivel de información acerca del mercado de valores con el que cuentan los directivos de las medianas y grandes empresas del departamento de Boyacá; debido al evidenciado rezago en lo que respecta a la apropiación por parte del empresariado Boyacense de los temas de toma de decisiones financieras, y en consecuencia en lo concerniente al sistema financiero específicamente el mercado de valores, con base en esto se pretende establecer panoramas que brinden herramientas de diagnóstico para la formulación de planes estratégicos tendientes al aprovechamiento de las oportunidades y ventajas que en términos financieros les brinda el mercado de valores a las diferentes organizaciones.

\section{PALABRAS CLAVE}

Mercado de valores, inversión, financiación, información, estrategia, medianas y grandes empresas

\begin{abstract}
The present study is approached from the analytical and strategic perspective in the area of corporate finance, seeking to determine the level of information about the market in that count managers of medium and large enterprises in Boyacá department due to evident lag with regard to the appropriation by the Boyacense entrepreneurship issues of financial decision making, and therefore specifically with regard to the stock market financial system, based on this set panoramas basis to provide diagnostic tools for formulation aimed at exploiting the opportunities and benefits financially gives the stock market to different organizations strategic plans.
\end{abstract}

\section{KEYWORDS}

Stock market, investment, financing, information, strategy, medium and large companies

\section{INTRODUCCIÓN}

Invertir o financiarse a través del banco es una opción sencilla y rápida; sin embargo, los tiempos cambian y se deben explorar nuevas opciones que les permitan a las empresas mayor flexibilidad y mejores tasas, estas opciones las brinda la participación en el mercado de valores.

Alrededor del mundo bursátil están la economía y las finanzas de una persona, una región, un sector industrial y un país. La globalización ha conseguido que los fenómenos de una región o país específico repercutan en todo el mundo. Antes no era imaginable que un país pudiera influir en las tasas y la paridad cambiaria de otro.

Aun cuando muchos de los empresarios crean no tener relación con el mercado de valores, cada vez se está más vinculado y la perspectiva vislumbra que la participación se acrecentará con el tiempo. "Un crecimiento sustancial ha logrado el mercado de valores en Colombia en los últimos años, no solo porque más inversionistas están 
CONOCIMIENTO DEL MERCADO DE VALORES EN LAS MEDIANAS Y GRANDES EMPRESAS DEL DEPARTAMENTO DE BOYACÁ: DIAGNÓSTICO Y PROPUESTA ESTRATÉGICA

llegando al sistema, sino porque la valorización de los activos ha sido elevada..." (González, 2010).

A medida que se incremente el ingreso nacional y se mantenga una menor tasa de inflación, aumentará la capacidad de ahorro, lo que obliga a buscar alternativas de inversión diferentes a las tradicionales, donde el mercado de valores será un actor importante.

Partiendo de esta base se evidencia la gran importancia que tiene para las empresas del departamento elegir mejores formas de financiamiento e inversión, para conseguir un escalonamiento en la competitividad regional y nacional. De allí que se pretende responder al cuestionamiento que se ha planteado acerca de cuál es el nivel de información de las empresas en cuanto al mercado de valores y de qué manera se han aprovechado las ventajas extraídas de dicho mercado, enfocándola al papel específico de las empresas del departamento de Boyacá y, partiendo de ello, formular un plan estratégico tendiente a vincular los resultados del diagnóstico de la situación actual con los lineamientos que permitan la convergencia hacia actividades de aprovechamiento financiero de las ventajas arrojadas por el aprovechamiento del Mercado de Valores.

\section{METODOLOGÍA}

Para el caso de la investigación se tomó como punto de partida, el diseño metodológico que se enuncia a continuación.

\section{Método de investigación}

Inductivo - Deductivo, pertenece a este tipo de investigación porque partiendo de las generalizaciones encontradas sobre conceptos, características, ventajas y oportunidades extraídas del mercado de valores se busca establecer particularidades acerca del nivel de información y conocimiento en cuanto a este mercado en las medianas y grandes empresas del departamento de Boyacá, y con ello lograr identificar y cuantificar las variables objeto de análisis, para finalmente permitir la formulación de un plan estratégico de acción tendiente a encaminar las actividades empresariales hacia la vinculación de la Inversión y Financiación a través del mercado de valores.

\section{Población y Muestra}

La investigación tomó como población universo a las empresas del departamento de Boyacá, que están debidamente registradas en cámara de comercio y correspondan a la clasificación de medianas y grandes de acuerdo con la ley 905 de 2004. Con las bases de datos de las cámaras de comercio de Tunja, Duitama y Sogamoso, se realizó el cálculo de una muestra representativa de acuerdo con los parámetros establecidos.

La muestra sobre la cual se realizan las encuestas objeto de análisis, se desarrolla para cada uno de los sectores del siguiente modo:

* Para el sector minero, subsector carbón se realizó la depuración de las bases de datos de acuerdo con las características de categorización de mediana o grande empresa, registro ante la Cámara de Comercio y similitud de la actividad económica, lo cual arrojó que existen en total 20 empresas 
correspondientes al subsector carbón en los municipios de Samacá, Socha y Sogamoso, sobre la totalidad de estas empresas se realizó la encuesta.

* Para el sector Industrial y Agroindustrial, se llevó a cabo la depuración de las bases de datos de acuerdo con las características de, categorización de mediana o grande empresa, registro ante la Cámara de Comercio y similitud de la actividad económica, lo cual arrojó la existencia de 68 empresas.

* Para el sector servicios, se realizó la depuración de las bases de datos de acuerdo con las características de, categorización de mediana o grande empresa, registro ante la Cámara de Comercio y similitud de la actividad económica, lo cual arrojó la existencia de 86 empresas.

* Para el sector Comercial se toma el total de empresas que figuran dentro de los parámetros establecidos, obteniendo un total de 17 empresas sobre las cuales se hizo la encuesta.

El estudio tuvo su soporte en una encuesta dirigida al dueño/administrador/gerente de las medianas y grandes empresas legalmente constituidas en el departamento de Boyacá y revisión de fuentes secundarias; para, además de realizar recolección de datos, identificar y relacionar variables como: el conocimiento del mercado de valores, disposición a la inversión, productos del mercado de valores en que puede participar cada empresa, perfil del inversionista y otras participaciones en el mercado de valores.

\section{Referentes Teóricos}

A continuación se presenta el marco teórico que siguió la investigación para su desarrollo, bajo unos fundamentos sólidos como etapa previa en el proceso investigativo.

\section{Inversión en el mercado de valores}

La Estructura de Capital de una empresa refleja la manera como ésta financia todos sus activos, ya sea con capital (patrimonio) o con deuda (pasivo). Dentro de esta estructura se encuentran implícitos las decisiones de inversión y financiamiento de la organización.

Como ya lo referían Franco Modigliani (nobel de economía 1985) y Merton Miller (Martínez y otros, 2007) (nobel de economía 1990), a finales de la década de los 50‘s, en la Teoría de la Estructura del Capital, dos empresas con activos idénticos, deben valer lo mismo; situación indiferente de su estructura en el pasivo o en el patrimonio.

"Dado que las decisiones de financiamiento no afectan el valor de la compañía, estas son completamente separables de las decisiones de inversión" (Martínez, Herazo y Corredor, 2007, p.111). Bajo ciertas condiciones como la ausencia de costos de quiebra, de impuestos y la existencia de uniformidad en la información de mercado, esta teoría argumenta que en el valor de la empresa no influye la conformación de su capital. Una organización empresarial puede funcionar, bien con los aportes de sus accionistas, así como con la emisión de deuda; es pertinente tener en cuenta otro de los supuestos de la teoría "a mayor endeudamiento, mayor riesgo corren los accionistas y más grande debe ser el rendimiento esperado por sus acciones" (Martínez y otros, 2007, p.112). El riesgo está ligado al costo de capital -WACC- 
CONOCIMIENTO DEL MERCADO DE VALORES EN LAS MEDIANAS Y GRANDES EMPRESAS DEL DEPARTAMENTO DE BOYACÁ: DIAGNÓSTICO Y PROPUESTA ESTRATÉGICA

que a su vez se relaciona con la clase de riesgo, dependiendo esto de las actividades operacionales de la empresa y siendo indiferente de la estructura de capital.

El riesgo es un factor importante en las decisiones de financiamiento y aún más influyente en las de inversión; este puede mitigarse teniendo en cuenta varias alternativas, una de ellas la diversificación.

En el libro Selección de Carteras: Diversificación eficiente, el nobel de economía (1990) Harry Markowitz (Martínez y otros, 2007), se expone acertadamente su Teoría del Portafolio, acerca de los modelos de inversión en carteras de acciones. Markowitz realiza su estudio en el marco de uno de los mercados más importantes a nivel mundial NYSE. La teoría expone la idea de que "los inversionistas construyen portafolios basados exclusivamente en el riesgo y en el rendimiento esperado". Medina (2003, p. 129), argumenta que la reducción del riesgo en una inversión es posible, si dicha inversión se distribuye entre dos o varios activos financieros, es decir construyendo un portafolio. Para ello realizó la aplicación de la herramienta matemática de programación lineal, determinando un área de posibilidades de la factibilidad, al relacionar el riesgo con el retorno (utilidad, ganancia), al trazar el área, se establecía lo que Markowitz llamó: frontera de inversiones, dentro de la cual se ubican las combinaciones que ofrecen alternativas para el inversionista.

Además del análisis básico propuesto en la teoría, Markowitz expone que se hacen necesarios: "el análisis de las empresas y de los sectores económicos, análisis de la interacción entre el riesgo y retorno de cartera, selección de la cartera y gestión de la cartera" (Martínez y otros, 2007, p.9).

Se establecen unos parámetros en el modelo, los cuales son: Riesgo-retorno: "simboliza que el riesgo de una cartera se divide en dos: por un lado, está el sistémico o no diversificable, (riesgo de mercado) y por el otro el riesgo no sistémico o diversificable (inherente a cada acto individual)" (Martínez, Herazo y Corredor, 2007, p.9). El primer riesgo, incluye aspectos como política económica y variables macroeconómicas, el segundo está más ligado a la misión de la empresa, su actuar directivo y la conformación de su estructura de capital.

Retorno-riesgo, establece la necesidad de la aplicación de herramientas que permitan al inversionista el cálculo del retorno esperado, así como las posibles volatilidades de ese retorno, para que este tenga argumentos de decisión.

Teniendo en cuenta los parámetros, Markowitz establece la aplicación de instrumentos matemáticos-estadísticos como el cálculo de la media ponderada de los retornos, el riesgo medido a través de la desviación estándar del activo y el coeficiente de correlación para los valores.

Igualmente, James Tobin, en 1958, en su teoría del portafolio y de la demanda del dinero, hace alusión también a evitar la no diversificación, Tobin desarrolló un modelo de demanda especulativa de dinero, donde se consideran diferentes activos financieros con distinto riesgo y rentabilidad, situación que propicia la diversificación. El inversionista debe escoger la mejor combinación de activos según sus características propias en cuanto a riqueza y preferencias.

En este contexto, se han establecido factores como el retorno, el riesgo-sistémico y no sistémico, pero existe otro aspecto que debe ser incluido como influyente en las decisiones de financiamiento e inversión tomadas en una organización empresarial: el ahorro. 
Al respecto el economista estadounidense y nobel de economía (1990) William Sharpe, para la década de los 60‘s, enfoca su Teoría de la Selección de Carteras y el Mercado de Capitales en Condiciones de Riesgo, hacia la gestión de los portafolios en mercados de capitales, orientados al ahorro-inversión. "La teoría de cartera se orienta hacia las predicciones que debe tener en cuenta un inversionista y como tomarlas en función de las expectativas propias y del mercado para seleccionar la mejor cartera de valores" (Martínez y otros, 2007). Lo más importante del modelo que diseño Sharpe es que plantea el cálculo de los valores tanto para contexto de incertidumbre como de certeza; utilizando datos reales mediante procedimientos estadísticos y econométricos.

La teoría demostró que los inversionistas están en capacidad de escoger portafolios diferentes, debido a que sus perspectivas en aspectos como el mercado, percepción del riesgo, capacidad de inversión y rentabilidad esperada, son distintas y están determinadas por características propias del individuo, o en el caso de la presente investigación, de la organización empresarial. La teoría expuesta por William Sharpe, fijo un punto de partida para el surgimiento de otra teoría: Capital Asset Pricing Model CAPM en el año de 1964, elaborada por Jack L Treynor, John Litner y Jan Mossin, quienes plantearon un modelo matemático que es utilizado para determinar la tasa de retorno teóricamente requerida para un cierto activo que es incluido en un portafolio de inversiones. El modelo toma en cuenta la sensibilidad del activo riesgo no diversificable (conocido como riesgo de mercado o riesgo sistemático) representado por el símbolo beta, así como también el retorno esperado del mercado y el retorno esperado de una activo teóricamente libre de riesgo.

Myron J. Gordon, se constituye como otro de los economistas relacionados con el mercado de valores. Es también teórico de administración de empresas, principalmente debido a su interés en la estimación del capital y del valor de las empresas. El modelo de Gordon, llamado Modelo de Dividendos Descontados con Crecimiento, está desarrollado alrededor del crecimiento de los dividendos en la empresa, este modelo se puede aplicar cuando una organización reinvierte las ganancias, que no deben haber sido paga das como dividendos "Si una compañía emite una nueva acción en los mercados financieros, incurre en un costo adicional. Las ganancias de la venta de la acción serán menores que el precio del mercado actual" (Keat y Young, 2004, p. 583), el modelo presupone un crecimiento de los dividendos a una tasa constante, siendo un modelo aconsejado para empresas con crecimiento bajo y constante a lo largo del tiempo (Black, 2011).

\section{VARIABLES DEL MERCADO DE VALORES}

\section{CONOCIMIENTO DEL MERCADO DE VALORES}

El conocimiento del mercado de valores se entiende como la posesión de datos acerca de su funcionamiento, entendido como la transacción de recursos de un sector superavitario (inversionistas) a un sector deficitario (empresas) y de su composición, comprendida por emisores de valores, inversionistas y facilitadores.

Es importante que el inversionista posea instrumentos e información general del mercado de valores en Colombia, para así facilitar la apropiada y orientada toma de decisiones de inversión, favoreciendo de este modo a la profundización y promoción de la participación en dicho mercado en el país. 
CONOCIMIENTO DEL MERCADO DE VALORES EN LAS MEDIANAS Y GRANDES EMPRESAS DEL DEPARTAMENTO DE BOYACÁ: DIAGNÓSTICO Y PROPUESTA ESTRATÉGICA

\section{Mecanismos de difusión del Mercado de valores}

a. SIMEV: Sistema Integral de Información del Mercado de Valores, "es el conjunto de recursos humanos, técnicos y de gestión administrados por la Superintendencia de Colombia para permitir y facilitar el suministro de información al mercado" (Superintendencia Financiera, 2006). El SIMEV se compone de los siguientes registros:

$$
\begin{aligned}
& \text { * } \quad \text { Registro Nacional de Valores y Emisores-RNVE. } \\
& \text { * } \quad \text { Registro Nacional de Agentes del Mercado de Valores-RNAMV. } \\
& \text { * Registro Nacional de Profesionales del Mercado de Valores-RNPMV. }
\end{aligned}
$$

b. Estudios técnicos: son informes estructurados y fundamentados que dan cuenta de diferentes aspectos del mercado de valores. Los más importantes son:

* Análisis Fundamental: técnica de análisis para predecir las cotizaciones futuras de un valor basada en un estudio minucioso de los estados contables de la empresa emisora, así como de sus expectativas futuras de expansión y de capacidad de generación de beneficios. Este análisis se basa en los datos concretos que afectan a una acción o cualquier otro activo. Los beneficios, las expectativas de tipos de interés, el endeudamiento, etc...Son los elementos que se barajan para tomar una decisión de compra o de venta sobre un activo financiero. (Bolsa de Valores, 2008)

* Análisis Técnico: Es la denominación aplicable a un conjunto de técnicas que tratan de predecir las cotizaciones bursátiles, teniendo en cuenta también el comportamiento de ciertas magnitudes bursátiles como volumen de contratación, cotización de las últimas sesiones, evolución de las cotizaciones en periodos más largos, capitalización bursátil, etc. El análisis técnico se apoya en la construcción de gráficos que indican la evolución histórica de los precios de los valores y en técnicas analíticas que pueden predecir las oscilaciones bursátiles. Se le denomina también análisis grafico o chartista, aunque el análisis técnico es más amplio porque incorpora modelos matemáticos, estadísticos y econométricos (Bolsa de Valores, 2008).

\section{Riesgos de participación en el Mercado de Valores}

a. Riesgo de mercado: implica la pérdida atribuida a cambios o variaciones en los precios de los activos o títulos en lo que se ha invertido.

b. Riesgo de crédito: es el riesgo de incumplimiento de pago de dividendos o de la obligación misma, por parte de la entidad emisora del título valor.

c. Riesgo de liquidez: es la dificultad o facilidad de compra-venta de un activo.

d. Riesgo legal: hace referencia a dificultades de tipo legal que puedan llegar a perturbar el veraz cumplimiento de las condiciones de la inversión previamente pactadas y que puedan vulnerar los derechos del inversionista. 
e. Riesgo operativo: derivado de fallas en los procesos, sistemas, modelos o equivocaciones humanas que pueden restarle valor a la inversión.

\section{DISPOSICIÓN PARA LA INVERSIÓN EN EL MERCADO DE VALORES}

\section{Condiciones para invertir en el Mercado de Valores}

a. Consideraciones preliminares: al tomar la decisión de invertir es importante tener en cuenta un término mencionado en los análisis financieros y en la diversificación. El inversionista debe tener en cuenta que al colocar su capital en varias inversiones diferentes va a minimizar el riesgo de que su patrimonio se vea afectado, a su vez va a tener mayores posibilidades de que todas sus inversiones sean rentables, mejorando el rendimiento promedio de su portafolio; por el contrario si se coloca todo el dinero en una sola inversión y por alguna razón esta no resulta favorable, todo su capital quedara expuesto y con el riesgo de perder.

Es necesario también tener en cuenta aspectos como: el monto o cantidad de dinero a invertir, el plazo o vencimiento de los títulos valores, la liquidez de los mismos que es la facilidad que presenta el titulo valor para venderse o comprarse en el mercado a un precio justo, la rentabilidad que es el mayor o menor grado de ganancia que puede obtener el inversionista por su dinero y el riesgo grado de certeza o incertidumbre acerca de la calidad de los títulos valores.

Por otro lado debe tenerse en cuenta que existen unas firmas, llamadas sociedades comisionistas de bolsa, quienes son los promotores de los negocios que están adscritos a la Bolsa de Valores de Colombia y tienen la facultad de ejecutar transacciones en el mercado de valores a título del inversionista. El objetivo principal de dichas organizaciones es el de asesorar y aconsejar al inversionista teniendo en cuenta su perfil, necesidades y expectativas. El asesor comisionista, inicialmente abre una cuenta a nombre del nuevo inversionista, empieza a realizar la transacción o transacciones que acordó con el mismo, simultáneamente registra la cuenta en un deposito central de valores, entidad que a su vez realiza el manejo electrónico de todas las operaciones.

Si el capital con el que cuenta es pequeño, tiene la posibilidad de pertenecer a una cartera colectiva, la cual conforman varios capitales de diferentes montos y así el inversionista puede lograr diversificación.

b. Requisitos generales: a continuación se presentan los requisitos, que en general, exige una sociedad comisionista de bolsa para la realización de una inversión, aunque pueden variar de acuerdo a la entidad.

* Un capital mínimo de un millón de pesos (\$1.000.000).

* Diligenciamiento de una carpeta de documentos con información personal.

* Diligenciamiento de una carpeta de documentos con información personal de un segundo titular. 
CONOCIMIENTO DEL MERCADO DE VALORES EN LAS MEDIANAS Y GRANDES EMPRESAS DEL DEPARTAMENTO DE BOYACÁ: DIAGNÓSTICO Y PROPUESTA ESTRATÉGICA

* Adjunto de copia de la última declaración de renta (en el caso de que el inversionista declare).

c. Protección al inversionista: en Colombia, las personas naturales y jurídicas cuentan con tres mecanismos de protección al inversionista. (i) Defensor del cliente: es un vocero que tiene como función conocer y resolver de la manera más objetiva, independiente y gratuita las quejas individuales, existe uno en cada sociedad comisionista de bolsa. (ii) Autorregulador del Mercado de Valores AMV: es el organismo de autorregulación, entre sus funciones esta prestar servicios de supervisión, disciplina y regulación del mercado, en aspectos como la compra venta, supervisión de cumplimiento de la normativa vigente mediante una gestión monitoreo del mercado en tiempo real, ejerce control sobre personas naturales, jurídicas e intermediarios del mercado de valores. (iii) Superintendencia financiera de Colombia: "Entidad cuyo objetivo es supervisar el sistema financiero colombiano con el fin de preservar su estabilidad, seguridad y confianza así como promover, organizar y desarrollar el mercado de valores colombiano y la protección de los inversionistas, ahorradores y asegurados" (Bolsa de valores de Colombia, 2008).

PRODUCTOS DEL MERCADO DE VALORES

\section{Renta Fija}

La renta fija se constituye en instrumentos que "son emisiones de deuda que realizan los estados y las empresas dirigidos al mercado" (Bolsa de valores de Colombia, 2008). Estas emisiones son hechas en cantidades previamente definidas, con fecha de vencimiento e intereses estipulados en el momento de su lanzamiento en el mercado primario. En el mercado Colombiano los principales títulos de renta fija son los siguientes:

a. Aceptaciones Bancarias: son letras de cambio en las cuales una entidad financiera actúa como aceptante y debe cumplir directamente las obligaciones respectivas.

b. CDT: son títulos valores emitidos por entidades financieras, las cuales reciben un capital y deben devolverlo al depositante en un plazo determinado. Para efectos de liquidez el depositante puede negociarlos en el mercado de valores, con el riesgo de recibir un poco menos de los rendimientos esperados, pero con la ventaja de obtener el dinero en el momento en el que lo necesita.

c. Tidis: Títulos de Devolución de Impuestos emitidos por el Ministerio de Hacienda y crédito público con el objetivo de que la Dian devuelva saldos a favor a los contribuyentes, por los Tidis no se emiten títulos físicos, poseen una vigencia de un año, no devengan intereses y solo servirán para cancelar impuestos en el año siguiente a la fecha de expedición.

d. TES: son los títulos de deuda pública que emite la Tesorería general de la nación, pueden ser en UVRs o indexados a la TRM, son una de las mayores fuentes de financiación del gobierno (ley 51 de 1990) y los subasta en Banco de la Republica, pueden ser negociados en el mercado secundario, además cuentan con una calificación AAA y están respaldados la nación.

e. Bonos: Son un préstamo de dinero que el inversionista le hace al empresario 
y por el cual recibirá un rendimiento en intereses, proporcional a la cantidad de dinero que aporta y al plazo del préstamo. Los bonos de deuda tienen una renta fija que se pacta al momento de la emisión y depende del plazo del préstamo, la calificación de la empresa se llama fija porque no depende del comportamiento económico de la empresa. (Bolsa de valores de Colombia, 2008)

\section{Renta Variable}

La renta variable es una parte del mercado que emite títulos valores "cuya rentabilidad depende de diversos factores ligados fundamentalmente a los beneficios y expectativas de la empresa que cotiza títulos de propiedad en una bolsa (emisor)" (Bolsa de valores de Colombia, 2008). Los principales títulos de renta variable son:

a. Acciones: son títulos valores que se pueden negociar tanto en el mercado primario como en el secundario y básicamente representan un porcentaje de participación en la propiedad de una organización empresarial que es la entidad emisora. Las clases de acciones son: (i) Acción ordinaria: conceden a su titular derechos económicos y no económicos en la participación en el capital de la empresa emisora. (ii) Acción preferencial: conceden a su titular prioridad en el pago de dividendos y en caso de disolución de la empresa, el reembolso del capital, no implican derecho a voto.

\section{Divisas}

"Comprende tanto los billetes de bancos extranjeros como los saldos bancarios denominados en moneda extranjera" (Bolsa de valores de Colombia, 2008). El mercado de divisas es un marco en donde entidades de diversa índole como individuos, empresas o bancos, realizan compra-venta de monedas extranjeras, por medio de mecanismos que ayudan a facilitar dichas operaciones.

\section{Derivados}

El mercado de derivados comprende aquellas operaciones financieras que posibilitan la compra o venta de activos, pactada en una fecha futura.

a. Futuros: el contrato de futuro es estandarizado, este obliga a las partes en la compra o venta de una cantidad previamente pactada de un activo, para efectuarse en una fecha futura y con un precio acordado anticipadamente; con este los inversionistas mitigan riesgos como las variaciones de precios y las especulaciones.

b. Opciones: el contrato de opción, es llamado así, debido a que el comprador adquiere el derecho, pero no se ve obligado a comprar o a vender el activo a un precio pactado en una fecha futura (Superintendencia financiera de Colombia, 2008)..

Cabe mencionar que en las bolsas de valores fueron creados unos organismos llamados cámaras de compensación (clearing houses), con el objetivo de mediar en las operaciones de derivados. Su función principal es actuar como intermediaria entre compradores y vendedores, de modo que las partes contratantes se obligan con la cámara de compensación, mitigando el riesgo de contraparte; por ende dicho organismo asume la responsabilidad de cumplimiento de la negociación y actúa como comprador para la parte vendedora y vendedor para la parte compradora.

\section{RESULTADOS}

Diagnóstico Sectorial Del Nivel De Conocimiento Acerca Del Mercado De Valores En Las Empresas Boyacenses 
CONOCIMIENTO DEL MERCADO DE VALORES EN LAS MEDIANAS Y GRANDES EMPRESAS DEL DEPARTAMENTO DE BOYACÁ: DIAGNÓSTICO Y PROPUESTA ESTRATÉGICA

A continuación se presentan los resultados de la fase de diagnóstico:

Cuadro 1. Diagnóstico sectorial del nivel de conocimiento acerca del mercado de valores

en

\begin{tabular}{|c|c|c|c|c|}
\hline Variable & $\begin{array}{l}\text { Sector minerol } \\
\text { subsector carbón }\end{array}$ & Sector comercial & $\begin{array}{l}\text { Sector industrial y } \\
\text { agroindustrial }\end{array}$ & Sector Servicios \\
\hline $\begin{array}{l}\text { Conocimiento } \\
\text { acerca del } \\
\text { mercado de } \\
\text { valores }\end{array}$ & 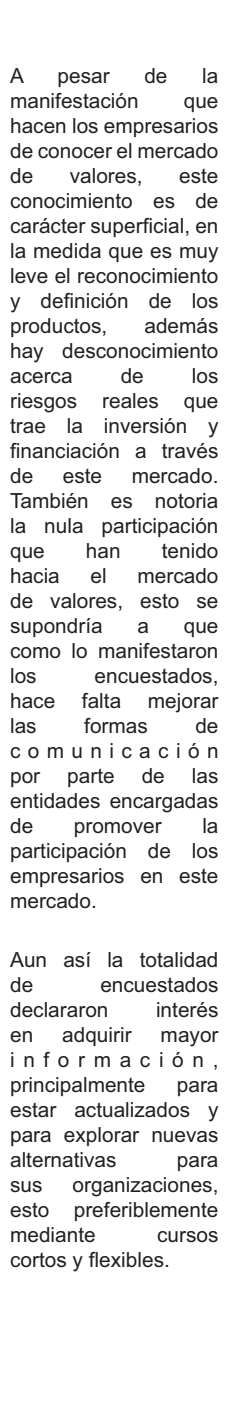 & 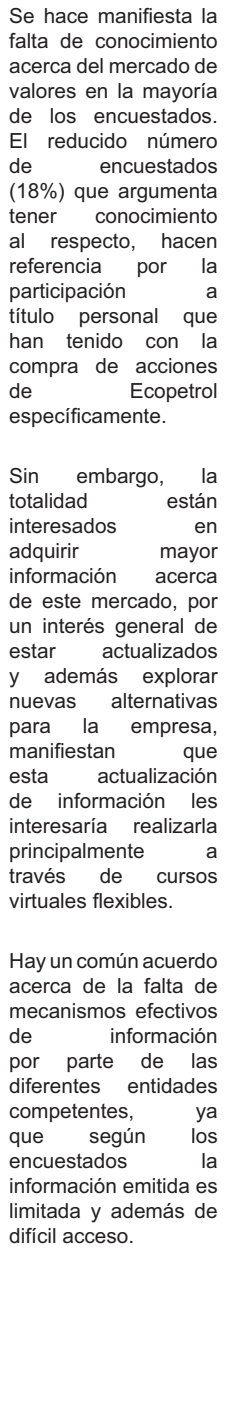 & 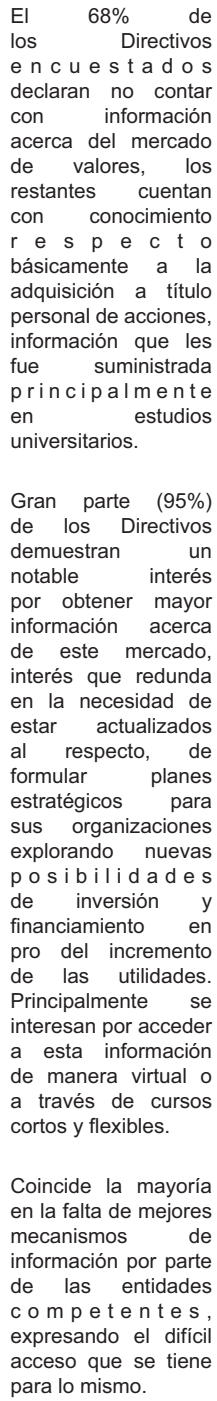 & $\begin{array}{l}\text { El desconocimiento de los } \\
\text { empresarios de este sector acerca } \\
\text { del mercado de valores es alto ya } \\
\text { que el } 92 \% \text { de los encuestados } \\
\text { manifiesta desconocimiento por } \\
\text { este mercado del mismo modo } \\
\text { el conocimiento de los riesgos } \\
\text { y condiciones de participar } \\
\text { en los mismos, esta situación } \\
\text { ocasiona que tanto el referente } \\
\text { conceptual que se tiene como } \\
\text { el conocimiento de instrumentos } \\
\text { de renta fija, renta variable, } \\
\text { derivados y divisas sea difícil de } \\
\text { estructurar para estas personas. } \\
\text { De otro lado la mayoría de } \\
\text { encuestados no sabe y responde } \\
\text { respecto a las motivaciones del } \\
\text { conocimiento del mercado de } \\
\text { valores y que dicha razón conlleve } \\
\text { a que se haya mostrado duda en } \\
\text { la disposición a obtener mayor } \\
\text { conocimiento de este mercado. } \\
\text { Se tiene entonces en este sentido } \\
\text { que el medio preferido para llegar } \\
\text { a un mayor conocimiento está } \\
\text { relacionado con medios como } \\
\text { el internet y los cursos cortos, } \\
\text { aunque se afirma que entidades } \\
\text { como la BVC, Asobancaria, } \\
\text { Superfinanciera no han creado } \\
\text { los mecanismos suficientes para } \\
\text { acceder a este mercado. }\end{array}$ \\
\hline
\end{tabular}




\begin{tabular}{|c|c|c|c|c|}
\hline $\begin{array}{c}\text { Disposición a } \\
\text { la inversión en } \\
\text { el mercado de } \\
\text { valores }\end{array}$ & $\begin{array}{l}\text { Los empresarios } \\
\text { e } \mathrm{n} \text { c u e s t a d o s } \\
\text { manifiestan tener } \\
\text { interés por la } \\
\text { inversión a través del } \\
\text { mercado de valores, } \\
\text { sin embargo, en su } \\
\text { mayoría desconocen } \\
\text { las condiciones para } \\
\text { realizar esta inversión } \\
\text { lo cual reafirma el } \\
\text { interés de los mismos } \\
\text { por complementar } \\
\text { su información } \\
\text { al respecto. } \\
\text { Básicamente se } \\
\text { inclinarían por adquirir } \\
\text { en el mercado de } \\
\text { renta fija, CDT's y en } \\
\text { el mercado de renta } \\
\text { variable, acciones. } \\
\text { Por otro lado les llama } \\
\text { la atención acceder al } \\
\text { mercado de divisas, } \\
\text { esto partiendo } \\
\text { de la base de la } \\
\text { exportación de carbón } \\
\text { y su negociación en } \\
\text { moneda extranjera. }\end{array}$ & \begin{tabular}{l} 
Tan solo la mitad \\
de los encuestados \\
desearían realizar \\
alguna clase de \\
inversión a través \\
del mercado de \\
valores, pero existe \\
un temor generalizado \\
debido esto al \\
desconocimiento \\
acerca de las \\
condiciones y los \\
diferentes riesgos que \\
trae. \\
\multicolumn{4}{c}{ Los interesados en } \\
realizar inversiones \\
futuras, se inclinan por \\
el aprovechamiento \\
del mercado de renta \\
fija, principalmente \\
por la seguridad que \\
brinda enfocándolo \\
a la adquisición \\
del mecanismo de \\
CDT's. Así mismo \\
se denota un interés \\
generalizado por \\
la adquisición de \\
acciones.
\end{tabular} & 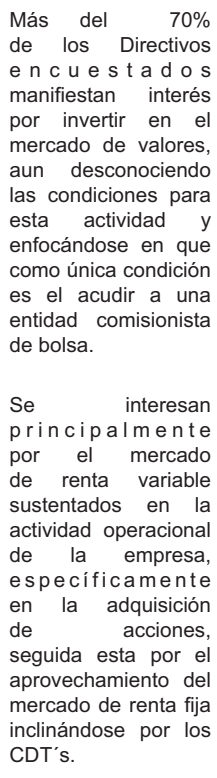 & $\begin{array}{l}\text { Teniendo en cuenta la falta de } \\
\text { conocimiento de los riesgos } \\
\text { relacionados con el mercado } \\
\text { de valores, los encuestados } \\
\text { mantienen una baja aceptación en } \\
\text { la participación en este mercado } \\
\text { con el } 36 \% \text { de las respuesta } \\
\text { favorables cuya preferencia } \\
\text { de inversión está enfocada a } \\
\text { la renta fija con el } 66 \% \text { de las } \\
\text { respuestas en lo que concierne } \\
\text { principalmente a CDT's con el } \\
55 \% \text {, }\end{array}$ \\
\hline $\begin{array}{c}\text { Perfil del } \\
\text { inversionista }\end{array}$ & $\begin{array}{l}\text { Al coincidir los } \\
\text { encuestados en que } \\
\text { invertirian en mayor } \\
\text { medida adquiriendo } \\
\text { acciones, se destaca } \\
\text { el perfil arriesgado. } \\
\text { El perfil conservador } \\
\text { se hace notorio } \\
\text { en menor medida } \\
\text { por la disposición } \\
\text { a la inversión en el } \\
\text { mercado de renta } \\
\text { fija. Por otra parte, en } \\
\text { cuanto al perfil neutro } \\
\text { hay un completo } \\
\text { desconocimiento de } \\
\text { éste por parte de los } \\
\text { encuestados. }\end{array}$ & $\begin{array}{l}\text { Este interés en Renta } \\
\text { fija y la inversión en } \\
\text { acciones, los hace } \\
\text { catalogarse en un } \\
\text { perfil de inversionista } \\
\text { neutro. } \\
\text { Seguido este del } \\
\text { perfil conservador } \\
\text { partiendo de la base } \\
\text { de la seguridad que } \\
\text { necesitan. El perfil } \\
\text { arriesgado está } \\
\text { categorizado en una } \\
\text { mínima proporción. }\end{array}$ & $\begin{array}{l}\text { Los empresarios } \\
\text { objeto de la encuesta } \\
\text { consideran su perfil } \\
\text { inversionista de } \\
\text { carácter conservador, } \\
\text { guiados por la } \\
\text { seguridad. Seguido } \\
\text { del perfil neutro y } \\
\text { finalmente en una } \\
\text { menor proporción, el } \\
\text { perfil arriesgado con } \\
\text { tan solo el } 9 \% \text {. }\end{array}$ & $\begin{array}{l}\text { Las personas encuestadas en } \\
\text { este sector económico enfocarían } \\
\text { sus esfuerzos a un perfil } \\
\text { inversionista neutro con el } 53 \% \\
\text { de las respuestas, seguido de un } \\
\text { perfil conservador con el } 46 \% \text { de } \\
\text { las respuestas. }\end{array}$ \\
\hline
\end{tabular}

Fuente: Elaboración propia 
CONOCIMIENTO DEL MERCADO DE VALORES EN LAS MEDIANAS Y GRANDES EMPRESAS DEL DEPARTAMENTO DE BOYACÁ: DIAGNÓSTICO Y PROPUESTA ESTRATÉGICA

Elementos estratégicos para el aprovechamiento de las oportunidades del mercado de valores

\section{La matriz DOFA del mercado de valores en el departamento de Boyacá}

Con el fin de llegar a determinar las estrategias que propendan el acercamiento de las empresas al mercado de valores, en mediano plazo, de acuerdo con los resultados obtenidos en el estudio, se propone un análisis DOFA, en el cuál se plantean las fortalezas, debilidades, oportunidades y amenazas, como se aprecia continuación:

Cuadro 2. Matriz DOFA del mercado de valores en Boyacá

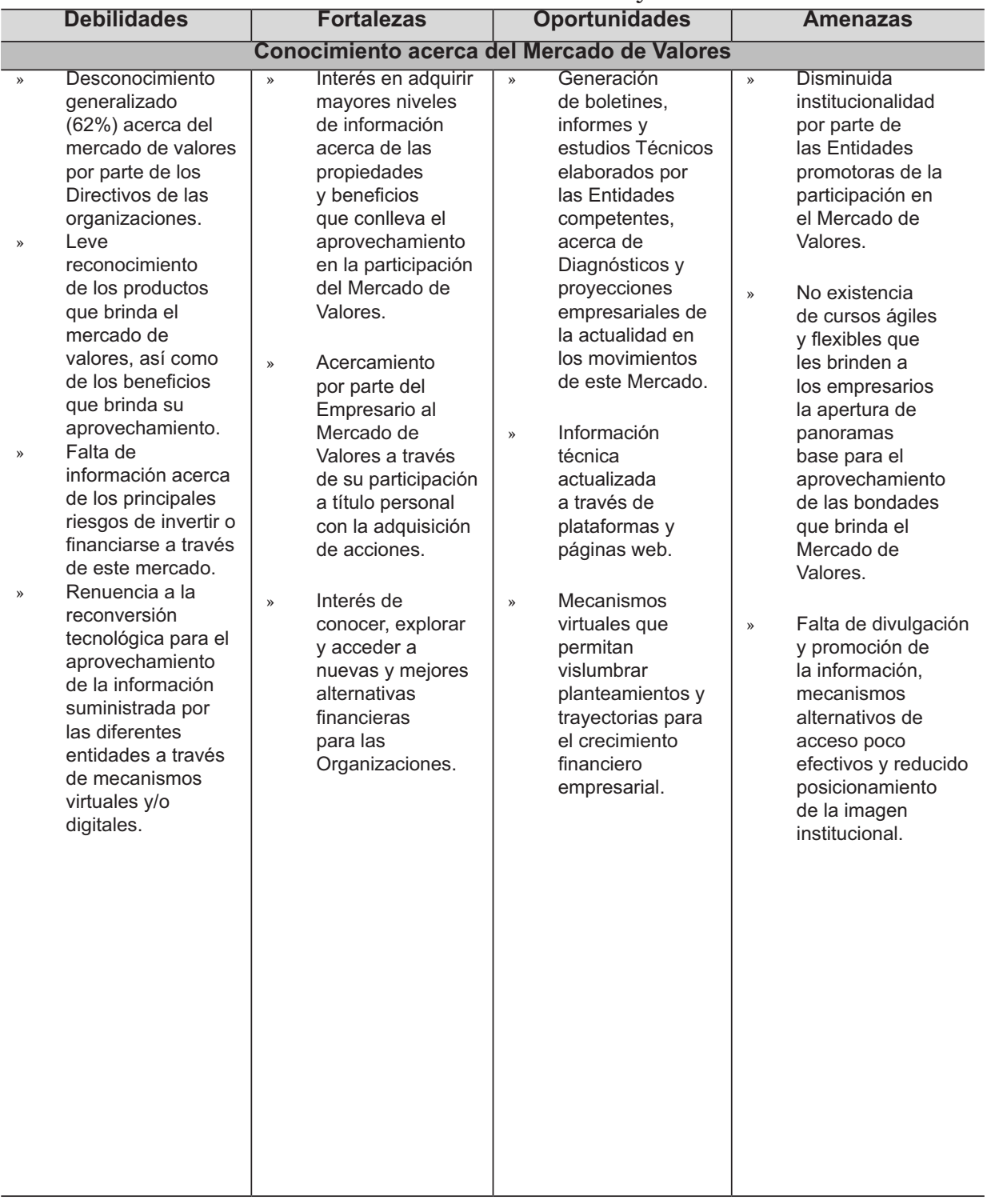




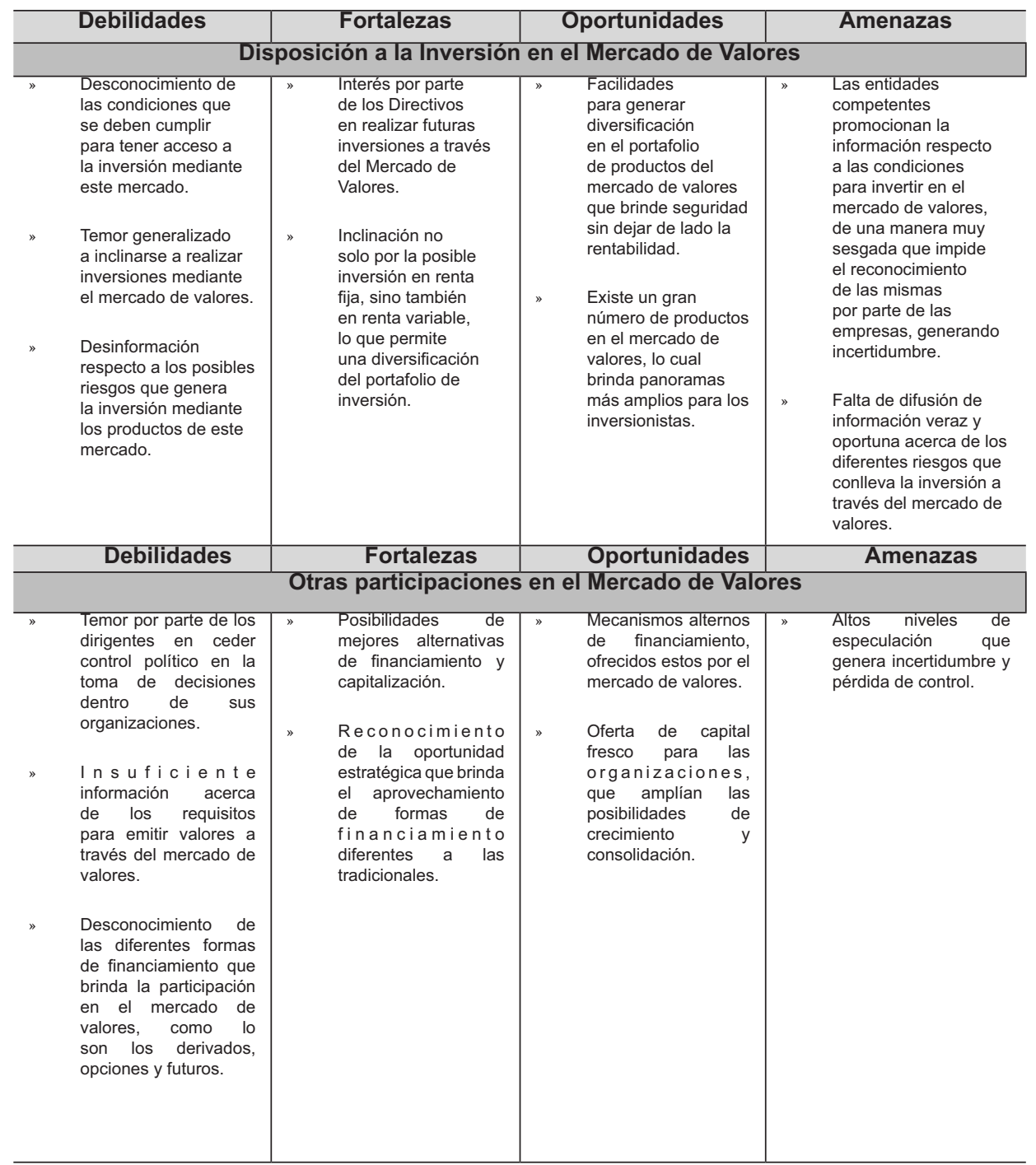

Fuente: Elaboración propia 
CONOCIMIENTO DEL MERCADO DE VALORES EN LAS MEDIANAS Y GRANDES EMPRESAS DEL DEPARTAMENTO DE BOYACÁ: DIAGNÓSTICO Y PROPUESTA ESTRATÉGICA

\section{Templo Estratégico Para El Aprovechamiento Del Mercado De Valores Por Parte De Los Empresarios Boyacenses}

La función de la planeación es un paso eminentemente prospectivo, es decir, que partiendo de un claro diagnóstico y la construcción de un escenario futuro deseado, busca definir las políticas, los objetivos, los factores claves de éxito, las grandes líneas de acción estratégica, los fundamentos que permitan trabajar en el hoy de forma efectiva, para alcanzar ese mañana visionado. Es así que con la conformación de un templo estratégico para el aprovechamiento del mercado de valores, se realiza una propuesta para el departamento de Boyacá.

Figura 1. Templo estratégico para el aprovechamiento del mercado de valores por parte de los empresarios Boyacenses

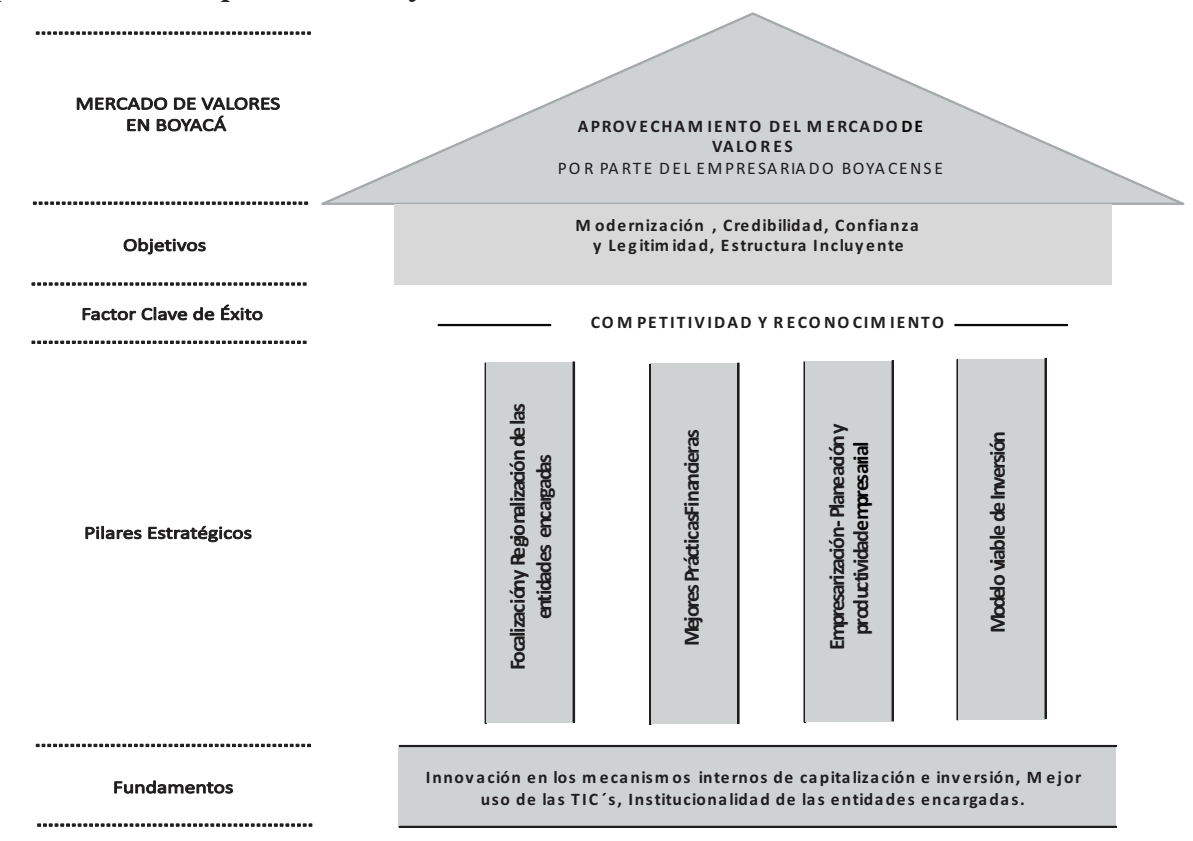

Fuente: Elaboración propia

\section{Los objetivos}

Modernización. Analizada ésta desde una institucionalidad del mercado de valores en el departamento de Boyacá de manera extendida y cohesionada alrededor de una entidad con capacidad, primero, para concebir la importancia de generar una mirada amplia por parte de los empresarios hacia las bondades que brinda este mercado, buscar una finalidad común, establecer metas, estrategias, acciones y realizar el debido seguimiento a las mismas; y segundo, para liderar este proceso desde la perspectiva de las responsabilidades para con los diferentes sectores, así mismo desde las que corresponden a agentes externos de la institucionalidad pública y privada. Esta y no otra es la articulación funcional de las Entidades como la Bolsa de Valores de Colombia, Autorregulador del Mercado de Valores, Superintendencia de Valores encargadas de fomentar el acercamiento empresarial 
hacia el mercado de valores, y para cumplirla requieren también de condiciones y acciones que apalanquen esa capacidad de convocatoria y de liderazgo en la acción.

Credibilidad, Confianza y Legitimidad. Se trata de objetivos altamente trascendentales para el proceso de modernización que inspira este Plan Estratégico, toda vez que la imagen distorsionada que se ha fundado en los Directivos de las empresas departamentales, sumado esto a los estereotipos y estigmas que recaen sobre el mismo, han tenido efecto negativo en el acercamiento y vinculación hacia el mercado de valores.

Permitir el surgimiento y reconocimiento de las entidades financieras del mercado de valores mediante la generación de credibilidad y confianza en los empresarios para captar su afiliación efectiva a las actividades bursátiles. Derribar los estereotipos que califican a este mercado como riesgoso, altamente complejo, y netamente desconocido por la comunidad.

Estructura Incluyente y Participativa. Hay una condición previa para lograr tan significativos niveles de apropiación efectiva, y se relaciona con generar la capacidad para entender las oportunidades que se pueden llegar a extraer al invertir y financiar las actividades de las organizaciones mediante el mercado de valores, y generar de esta manera una estructura institucional incluyente y participativa.

Incluyente en la medida que se le brinde a las organizaciones la oportunidad de acceder a los mecanismos de conocimiento que propendan porque la población de medianas y grandes empresas gestadas en el departamento de Boyacá incluyan en su imaginario formas alternas de financiación y de inversión diferentes al mercado intermediado, como el caso del mercado público de valores. De la misma forma en un mediano plazo se profundice, entendido este como el mayor desarrollo del mercado de valores medido en forma conjunta por el tamaño, la liquidez, la eficiencia y la concentración del mercado, especialmente hacia las medianas empresas.

Participativo, en la medida que las condiciones de entrada a este mercado brinden la posibilidad del desarrollo de un segundo mercado en el que estén insertas las medianas empresas, con el fin de generar oportunidades de crecimiento, desarrollo y apalancamiento empresarial y la consecuente ampliación en el número de empresas listadas en la bolsa de valores.

\section{Fundamentos}

Innovación en los mecanismos internos de capitalización e inversión. Es recurrente en la actualidad que las medianas y grandes empresas del departamento de Boyacá, estén financiadas principalmente a través del mercado intermediado (Bancario), dicha situación ocasiona que no hagan uso de formas alternas de financiamiento y de inversión que posiblemente generen menores costos de oportunidad y de esta manera profundicen en sus estrategias competitivas. Es así que, una de las innovaciones para las empresas catalogadas como medianas, en relación a su estructura de capital, sea la vinculación al mercado público de valores desde el punto de vista de la financiación, mientras que de otro la inclusión de dichas alternativas propendan por la generación de diferenciación desde el punto de vista de la estructura de inversión.

Mejor uso de las TIC's. Debido a la masificación del uso de las Tecnologías de la Información en los ambientes educativos y de las ventajas empresariales que conlleva la operacionalización de los mismos, se hace imprescindible que los directivos de estas organizaciones incluyan como parte de sus labores cotidianas $\mathrm{y}$, de procesos decisorios esta clase de herramientas en la medida que les surta 
CONOCIMIENTO DEL MERCADO DE VALORES EN LAS MEDIANAS Y GRANDES EMPRESAS DEL DEPARTAMENTO DE BOYACÁ: DIAGNÓSTICO Y PROPUESTA ESTRATÉGICA

de información oportuna, clave y precisa para generar unas bases teóricas sólidas tendientes a disminuir la incertidumbre que en la actualidad se evidencia por parte de los directivos, en cuanto a los riesgos, condiciones y requisitos para hacer parte del mercado de valores.

Institucionalidad de las entidades encargadas. Con relación con la Ley 1328 de 2009, en cuyos principios orientadores se establece la educación para el consumidor financiero de las entidades vigiladas por parte de la Superintendencia Financiera, es así como las instituciones que conciernen a este estudio han venido desarrollando, una gama de opciones educativas entre las que se evidencian en los puntos de la Bolsa de Valores de Colombia, cursos básicos para principiantes, cursos, programas y concursos con estudiantes universitarios y cursos avanzados E-trading, seminario y certificación AMV (2008) para expertos, cuyo fin es educar al consumidor financiero. Sin embargo, es evidente el desconocimiento por parte de los empresarios de la existencia de estas herramientas, de allí que sea latente el no aprovechamiento de dichos mecanismos y se haga necesario el posicionamiento institucional de estas entidades a través de estrategias de promoción.

\section{Pilares Estratégicos}

Focalización y regionalización de las entidades encargadas. La entidad que tiene mayor relevancia en el mercado público de valores, para el caso, la Bolsa de Valores de Colombia ha optado por una estrategia de descentralización en la que se tienen 30 puntos a nivel nacional, aun así, el departamento de Boyacá no cuenta con la oportunidad de hacer parte de este selecto grupo, siendo un departamento limítrofe con la capital del país, lo que genera un menor vínculo entre la Bolsa de Valores y el empresariado Boyacense.

Mejores Prácticas financieras. El cambio cada vez más constante del entorno está exigiendo que cada día las organizaciones adapten internamente acciones tendientes al aprovechamiento de las oportunidades que se prevén, la vinculación y crecimiento de sus fortalezas, el gestionamiento para la reducción de sus debilidades y el accionar estratégico para disminuir el impacto de sus amenazas.

Es así como han de determinarse las políticas corporativas que guiarán el cotidiano de las operaciones de la empresa, políticas que a su vez se constituyen en un aliciente para la formulación de estrategias de diferenciación competitiva y de esta manera sobrevivir a la exigencia del mercado. Dentro de estas políticas un factor clave es la toma de decisiones acertadas en el ámbito financiero, decisiones que giran en torno a la elección de alternativas mucho más rentables que les brinden a las organizaciones estabilidad, flexibilidad, retorno de la inversión, margen de oportunidad y capitalización, mismas que ofrece en su totalidad el mercado de valores.

Empresarización - planeación y productividad empresarial. Partiendo del enfoque de la eficiencia y la calidad total, ya producir al máximo con menos recursos no es suficiente, sino que, además, ha empezado a cobrar mucha más importancia la satisfacción del consumidor, el respeto por la naturaleza y lo más importante, producir lo que demandan los mercados, es decir, los consumidores en todo el mundo.

En la actual sociedad global del conocimiento, a la innovación, la Ciencia y la Tecnología les corresponde un papel fundamental para que el empresariado alcance y mantenga una capacidad permanente de incorporación en las últimas tecnologías, 
como factor de éxito de la Productividad y como única forma de posibilitar el logro de los altos niveles de competitividad que se requieren.

Modelo viable de inversión. Toda actividad económica requiere del apalancamiento financiero para promover su desarrollo. Solo mediante la inversión puede promoverse el crecimiento empresarial. La utilización de recursos financieros es esencial entonces, para promover cambios en la productividad vía inversión, o para posibilitar el sostenimiento y los deseables aumentos del volumen de la producción, mediante la inyección de capital de trabajo.

De ahí que sea altamente relevante que las empresas del departamento adecúen a sus actividades modelos internos que se conviertan en atractivos para los posibles inversionistas, modelos flexibles y rentables que amplíen el rango de potenciales lentes de inversión.

\section{CONCLUSIONES}

Más que una conclusión, esta es una invitación, primero, a no perder de vista la gran relevancia que para cualquier región simboliza el crecimiento empresarial y el auge de las oportunidades que esto genera; segundo, a vislumbrar las posibilidades que en el marco de la globalización tiene el apropiarse de la mejor manera de las bondades que simboliza el aprovechamiento del mercado de valores; y tercero, generar compromiso por la sostenibilidad, productividad y competitividad del empresariado Boyacense.

Es una invitación, además, que no solo se formula a los empresarios del departamento, sino también a todas aquellas organizaciones, públicas y privadas, que tienen alguna responsabilidad con la vinculación hacia el mercado de valores.

Los dirigentes, sin lugar a dudas, deberán hacer lo propio para lograr metas que también son comunes; y la institucionalidad de las entidades encargadas deberán comprometerse con la expansión de una imagen clara, y con la generación de las condiciones para el desarrollo frente a los retos competitivos, sin lo cual los

esfuerzos del empresariado, se verán prácticamente neutralizados. 
CONOCIMIENTO DEL MERCADO DE VALORES EN LAS MEDIANAS Y GRANDES EMPRESAS DEL DEPARTAMENTO DE BOYACÁ: DIAGNÓSTICO Y PROPUESTA ESTRATÉGICA

\section{REFERENCIAS BIBLIOGRÁFICAS}

AMV (2008). "La dinamización del mercado del mercado de valores en Colombia: oferta y demanda”. Recuperado (11/07/2013) de http://www.amvcolombia.org.co/ commons/downloads.php?f=../attachments/data/ 20090211162008.pdf\&n=la\%20 dinamizaci\%F3n\%20del\%20mercado\%20de\%20valores\%20en\%20colombia:\%20 oferta $\% 20 y \% 20 d e m a n d a$.

Black, B. (2011). "Requisitos legales e institucionales para el establecimiento de un mercado de valores sólido”. Revista del Mercado de Valores. No. 2 Recuperado (02/07/2013) de http:/www.amvcolombia.org.co/attachments/data/Book_ Revista Analisis_2_web.pdf.

Bolsa de valores de Colombia. (2008). "Home.Inversionistas.Conceptos fundamentales". Bogotá. Recuperado (09/07/2013) de http://www.BVC.com.co/pps/ tibco/portalBVC/Home/Inversionistas/Conceptos + Fundamentales?action $=$ dummy

González, F. (21 de diciembre de 2010). "Mercado de valores se duplicó en tres años; expertos afirman que emisiones corporativas seguirán al alza”. Portafolio.co: Portal de Economía. de Recuperado 05/03/2013 de http:/www.portafolio.co/economia/elmercado-valores-se-duplico-tres-anos $>$

Keat, G. y Young, K. (2004) Economía de empresa. México D.F.: 4 ed. edit. Pearson Prentice Hall.

Martínez A., Herazo, C. y Corredor, V. (2007). Estado del Arte de las Finanzas. Bogotá: Universidad Santo Tomas Editorial y Publicaciones.

Medina, L. (2003). Aplicación de la Teoría del Portafolio en el Mercado Accionario Colombiano. Bogotá.

Superintendencia financiera de Colombia. (2006). SIMEV Recuperado (11/07/2013) de http://www.superfinanciera.gov.co/

Superintendencia financiera de Colombia. (2008). "Conceptos básicos del mercado de valores”. Bogotá: Superfinanciera. Recuperado (28/06/2013) de http://www. super financiera.gov.co/ConsumidorFinanciero/conceptosbasicosmv.pdf. 